\title{
BMJ Open Exclusion of patients with concomitant chronic conditions in ongoing randomised controlled trials targeting 10 common chronic conditions and registered at ClinicalTrials.gov: a systematic review of registration details
}

\author{
Céline Buffel du Vaure, ${ }^{1,2,3}$ Agnès Dechartres, ${ }^{2,3,4,5}$ Constance Battin, ${ }^{3}$ \\ Philippe Ravaud, 2,3,4,5,6 Isabelle Boutron ${ }^{2,3,4,5}$
}

To cite: Buffel du Vaure $C$, Dechartres A, Battin C, et al. Exclusion of patients with concomitant chronic conditions in ongoing randomised controlled trials targeting 10 common chronic conditions and registered at ClinicalTrials.gov: a systematic review of registration details. $B M J$ Open 2016;6:e012265 doi:10.1136/bmjopen-2016012265

- Prepublication history and additional material is available. To view please visit the journal (http://dx.doi.org/ 10.1136/bmjopen-2016012265).

Received 16 April 2016 Revised 2 September 2016 Accepted 6 September 2016

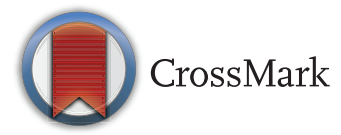

For numbered affiliations see end of article.

Correspondence to Dr Céline Buffel du Vaure; celine.buffel@aphp.fr

\section{ABSTRACT}

Objectives: To systematically assess registration details of ongoing randomised controlled trials (RCTs) targeting 10 common chronic conditions and registered at ClinicalTrials.gov and to determine the prevalence of (1) trial records excluding patients with concomitant chronic condition(s) and (2) those specifically targeting patients with concomitant chronic conditions.

Design: Systematic review of trial registration records. Data sources: ClinicalTrials.gov register.

Study selection: All ongoing RCTs registered from 1 January 2014 to 31 January 2015 that assessed an intervention targeting adults with coronary heart disease (CHD), hypertension, heart failure, stroke/transient ischaemic attack, atrial fibrillation, type 2 diabetes, chronic obstructive pulmonary disease, painful condition, depression and dementia with a target sample size $\geq 100$.

Data extraction: From the trial registration records, 2 researchers independently recorded the trial characteristics and the number of exclusion criteria and determined whether patients with concomitant chronic conditions were excluded or specifically targeted.

Results: Among 319 ongoing RCTs, despite the high prevalence of the concomitant chronic conditions, patients with these conditions were excluded in 251 trials $(79 \%)$. For example, although $91 \%$ of patients with CHD had a concomitant chronic condition, $69 \%$ of trials targeting such patients excluded patients with concomitant chronic condition(s). When considering the co-occurrence of 2 chronic conditions, $31 \%$ of patients with chronic pain also had depression, but $58 \%$ of the trials targeting patients with chronic pain excluded patients with depression. Only 37 trials $(12 \%)$ assessed interventions specifically targeting patients with concomitant chronic conditions; 31 (84\%) excluded patients with concomitant chronic condition(s).

Conclusions: Despite widespread multimorbidity, more than three-quarters of ongoing trials assessing
Strengths and limitations of this study

This is the first study assessing the exclusion of patients with concomitant chronic conditions from ongoing randomised controlled trials (RCTs) registered in ClinicalTrials.gov and assessing interventions for patients with common chronic conditions.

- We systematically assessed exclusion criteria reported in trial registration records of RCTs assessing interventions for patients with common chronic conditions and registered in ClinicalTrials.gov according to the prevalence of the co-occurrence of chronic conditions.

- Results may not be completely consistent with the exclusion criteria reported in the trial protocol and may underestimate the prevalence of excluding patients with concomitant chronic conditions in trials.

- The characteristics of patients actually included in these trials were not assessed. Some patients with concomitant chronic conditions may be excluded by investigators even if no exclusion criterion was listed in the protocol.

interventions for patients with chronic conditions excluded patients with concomitant chronic conditions.

\section{BACKGROUND}

Non-communicable chronic conditions are major public health challenges. ${ }^{1}$ Multimorbidity, defined as the coexistence of chronic conditions, is becoming the norm in primary care settings. ${ }^{2}{ }^{3}$ Its prevalence is increasing and represents $23 \%$ in the general population; that is, one in four adults is affected. ${ }^{4}$ Overall, more than half of patients with a 
chronic condition have multimorbidity ${ }^{5}$ and have an average of three chronic conditions. ${ }^{6}$ In the primary care setting, only $2.8-25.4 \%$ patients with a common chronic condition have no other chronic conditions. ${ }^{5}$

Despite this high prevalence of multimorbidity, clinical practice guidelines mainly focus on single chronic conditions and are not relevant for people with multiple chronic conditions. ${ }^{7-10}$ Indeed, polypharmacy (ie, the chronic coprescription of several drugs) is often the consequence of applying disease-specific guidelines to patients with concomitant chronic conditions. One consequence of polypharmacy is the high rate of adverse drug reactions, mainly from drug-drug interactions, which increase with the number of coexisting diseases and number of drugs prescribed. ${ }^{10}$ To develop appropriate guidelines for these patients, researchers need highquality evidence for patients with concomitant chronic conditions, particularly from randomised controlled trials (RCTs). Although we can never have good evidence for every possible combination of conditions, ${ }^{11}$ RCTs assessing treatment for a specific chronic condition should not exclude patients with concomitant chronic conditions.

This study examined ongoing RCTs targeting 10 common chronic conditions and registered at ClinicalTrials.gov. We assessed the prevalence of trial records excluding patients with concomitant chronic condition(s) and those specifically targeting patients with concomitant chronic conditions.

\section{METHODS}

We performed a systematic review of records of trials registered at ClinicalTrials.gov and assessing an intervention targeting patients with chronic conditions.

\section{Selection of chronic conditions}

We focused on 10 common chronic conditions frequently involved in multimorbidity in primary care settings: $:^{5}$ coronary heart disease (CHD), hypertension, heart failure, stroke/transient ischaemic attack (TIA), atrial fibrillation, type 2 diabetes, chronic obstructive pulmonary disease (COPD), painful condition, depression and dementia. We chose these 10 conditions because they are frequent and are frequently associated with concomitant chronic conditions. For instance, only $8.8 \%$ of the patients with CHD do not have an associated comorbid condition, $21.9 \%$ of those with hypertension, $2.8 \%$ with heart failure, $6.0 \%$ with stroke/TIA, $6.5 \%$ with atrial fibrillation, $17.6 \%$ with diabetes, $14.3 \%$ with COPD, $12.7 \%$ with painful condition, $25.4 \%$ with depression and $5.3 \%$ with dementia. ${ }^{5}$ For each of the selected conditions, we used the prevalence of their co-occurrence obtained from UK primary-care electronic health records from a study of the prevalence of multimorbidity in 1.75 million people. ${ }^{5}{ }^{6}$

\section{Identification of ongoing RCTs}

We identified all ongoing RCTs registered at ClinicalTrials.gov from 1 January 2014 to 31 January 2015 and assessing an intervention targeting patients with 1 of the 10 selected chronic conditions.

\section{Search strategy}

We searched ClinicalTrials.gov on 4 March 2015, using the key words 'coronary heart disease', 'hypertension', 'heart failure', 'stroke', 'transient ischemic attack', 'atrial fibrillation', 'diabetes', 'chronic obstructive pulmonary disease', 'chronic pain', 'depression' and 'dementia' in the Conditions field; 'recruiting' in the Recruitment field; 'Interventional studies' for Study types; 'adult (18-65)' and 'senior (66+)' for the Age group; with no restriction on country; and 'from 1 January 2014 to 31 January $2015^{\prime}$ in the First received field (ie, the date that summary clinical study protocol information was first submitted to the ClinicalTrials.gov registry). We exported all $\mathrm{xml}$ records from ClinicalTrials.gov and managed them by using R V.3.0.1 (Team RC. R: a language and environment for statistical computing. Vienna, Austria: R Foundation for Statistical Computing 2012) with the XML package.

\section{Study selection}

Two independent researchers systematically screened the retrieved records to include all trials assessing an intervention for patients with 1 of the 10 chronic health conditions as a stable chronic condition. Exclusion criteria were phase 0 , I and II studies and a target sample size $<100$ to limit the number of exploratory studies that usually have narrow eligibility criteria. Records were also screened to exclude cost-effectiveness studies, pharmacodynamic and pharmacokinetic studies, diagnostic studies, development of prediction rules and studies including a specific population (ie, palliative, peripartum and postpartum care).

\section{Data collection}

Some data were exported from ClinicalTrials.gov (ie, expected sample size, type of intervention, number of arms); type of outcome and eligibility criteria were recorded and classified by two independent researchers. Any disagreements were resolved by discussion to reach consensus or by arbitration with a third researcher.

\section{General characteristics of the studies}

From the records downloaded, we obtained the type of intervention (ie, drugs, device, surgery, behaviour, other), funding source, number of arms and target sample size. The primary outcomes were collected from the Primary Outcome Measures section of ClinicalTrials. gov and were classified as patient-important, surrogate and physiological or laboratory outcomes according to Gandhi $e t a l{ }^{12}$ A patient-important outcome was defined as 'a characteristic or variable that reflects how a patient feels, functions, or survives' (ie, all outcomes leading to important changes for patient life). ${ }^{13}$ 
Exclusion criteria

For each trial record, the two researchers independently screened the 'Eligibility' section. We recorded the number of exclusion criteria and determined whether the trial excluded patients with any concomitant chronic condition(s) (ie, 1 of the 10 chronic conditions selected or any other concomitant chronic condition). Chronic conditions that were not 1 of the 10 chronic conditions considered were classified as 'any other concomitant chronic condition'. When the chronic condition listed in the exclusion criteria was not clearly defined and could include one of the chronic conditions selected, we considered that the selected chronic condition was listed and that the study excluded patients with these chronic conditions. For example, when psychiatric disorder was listed among the exclusion criteria, we considered that patients with depression were excluded. When an acute stage of the selected chronic conditions was listed in the exclusion criteria, we did not consider that patients with this chronic condition were excluded.

\section{Trials specifically dedicated to patients with concomitant chronic conditions}

Researchers screened and recorded the chronic conditions targeted and determined whether the studies specifically included patients with $\geq 2$ chronic conditions.

\section{Statistical analysis}

Descriptive data are presented as number (percentage) or median (range) for categorical variables and mean (SD) for continuous variables. All analyses involved use of R V.3.0.1 (Team RC. R Foundation for Statistical Computing 2012).

\section{RESULTS}

\section{Study selection and characteristics}

Among the 1612 records retrieved from ClinicalTrials. gov, 319 for ongoing RCTs were selected (figure 1). These trials represented a total target sample size of 237544 patients. The RCTs targeted CHD in $36(11 \%)$ trials, hypertension in 38 (12\%), heart failure in 36 $(11 \%)$, stroke/TIA in $22(7 \%)$, atrial fibrillation in 25 $(8 \%)$, diabetes in $109(34 \%)$, COPD in $34(11 \%)$, chronic pain in $12(4 \%)$, depression in $40(13 \%)$ and dementia in $7(2 \%)$ (table 1). Interventions assessed were pharmacological in 147 trials (46.1), and 107 $(34 \%)$ were funded by industry. Outcomes were classified as patient-important outcomes in 199 (62\%) trials.

\section{Exclusion criteria}

Overall, 251 RCTs $(79 \%)$ excluded patients with concomitant chronic condition(s) (table 2 and figure 2), with a median of 9 (range 6-14) excluded chronic conditions per trial. Figure 3 shows, for each chronic condition, the proportion of trials excluding patients with concomitant chronic condition(s) by the prevalence of concomitant chronic conditions. For example, although
1612 records identified through Clinicaltrials.gov, screened and assessed for eligibility

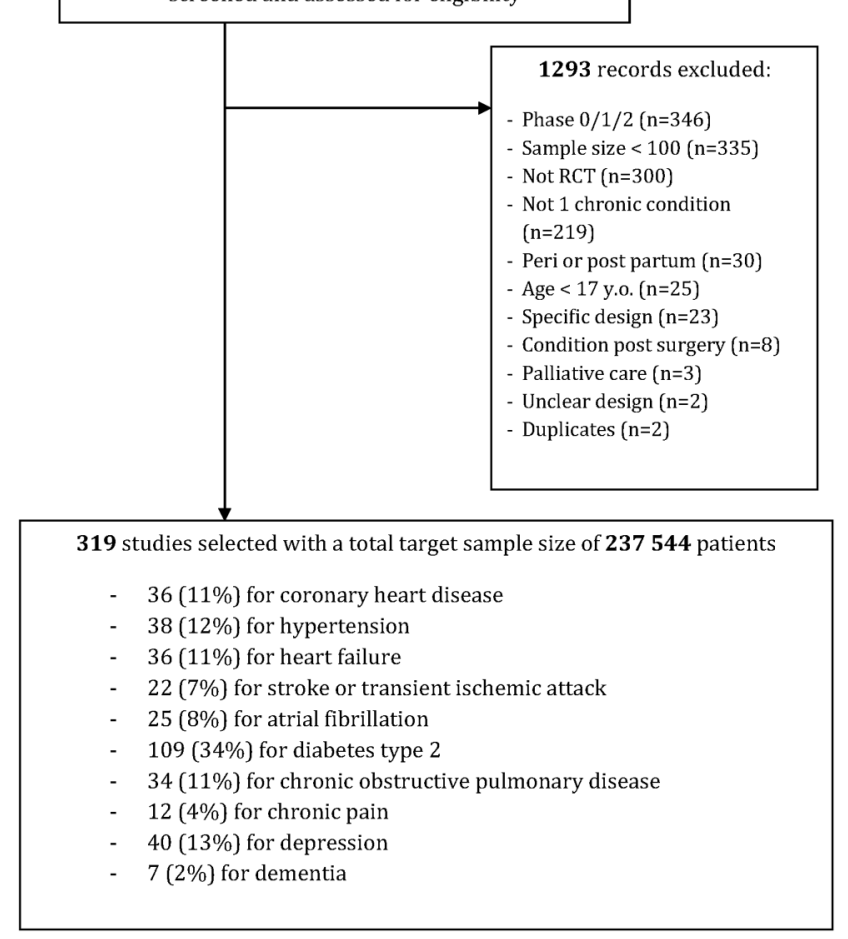

Figure 1 Flow chart of the selection process.

91\% of patients with CHD had a concomitant chronic condition, $69 \%$ of trials targeting patients with CHD excluded patients with concomitant chronic condition(s). Similarly, $97 \%$ of patients with heart failure had a concomitant chronic condition, but $81 \%$ of trials targeting patients with heart failure excluded patients with concomitant chronic condition(s).

Figure 4 reports the results when considering the prevalence of the co-occurrence of 2 chronic conditions among the 10 selected and shows variation according to the chronic conditions selected. For example, $31 \%$ of patients with chronic pain also had depression, but $58 \%$ of trials targeting patients with chronic pain excluded patients with depression. Overall, $41 \%$ and $32 \%$ of patients with dementia had hypertension or depression, respectively, but $43 \%$ of trials targeting dementia excluded patients with hypertension or depression.

\section{Trials specifically focused on patients with concomitant} chronic conditions

Only 37 trials (12\%) specifically assessed interventions for patients with $\geq 2$ chronic conditions (figure 2): 34 targeted patients with 2 chronic conditions; 2 targeted patients with 3 chronic conditions and 1 targeted patients with 4 chronic conditions. These 37 trials represent a total target sample size of $17708(8 \%)$ patients. Patients with diabetes mellitus were included in 14 trials (38\%), hypertension in 12 (32\%), CHD in 11 (30\%), heart failure in $7(19 \%)$, atrial fibrillation in $8(22 \%)$, depression in 7 (19\%) and stroke/TIA, chronic pain, 


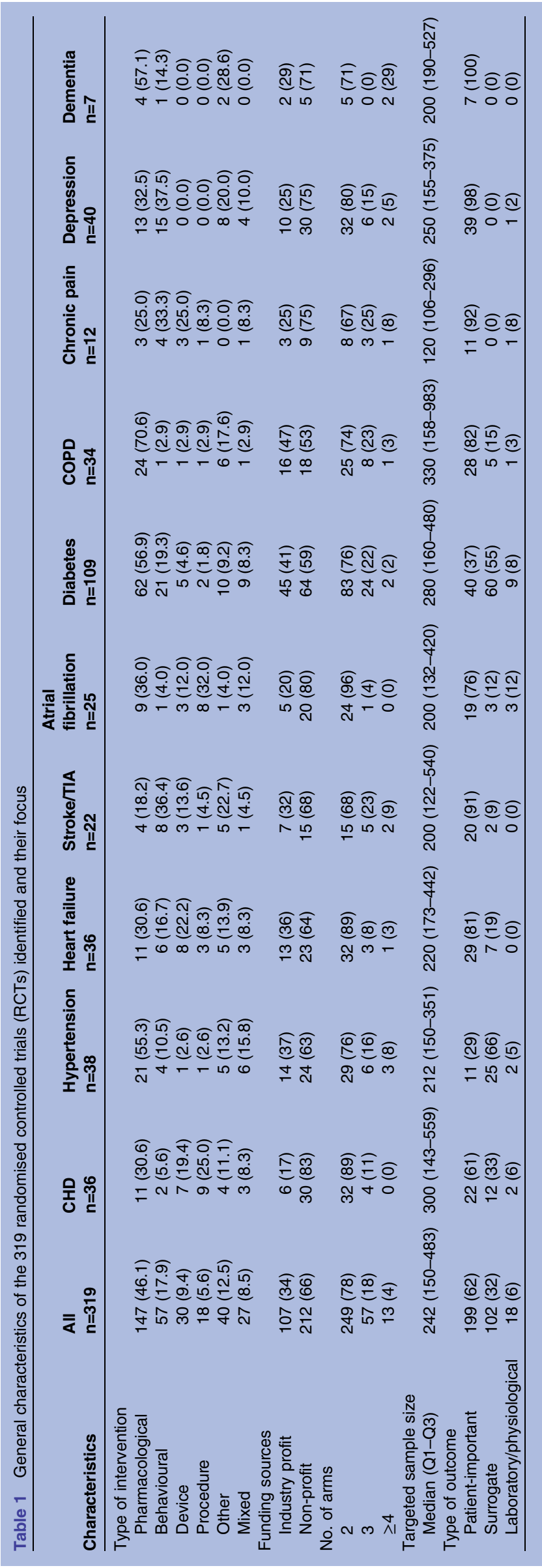

dementia and COPD in 1 trial (see online supplementary appendix 1). However, among the 37 trials, 31 (84\%) excluded patients with concomitant chronic conditions. Furthermore, the trials did not target several prevalent associations of chronic conditions.

\section{DISCUSSION}

This study reports how patients with concomitant chronic conditions are excluded from ongoing RCTs targeting patients with 1 of the 10 chronic conditions frequently involved in multimorbidity. Among 319 ongoing RCTs identified, 251 (79\%) excluded patients with concomitant chronic conditions, and the exclusion varied according to the chronic condition targeted. Only 37 trials (12\%) assessed interventions specifically targeted to patients with concomitant chronic conditions, and 31 (84\%) excluded patients with concomitant chronic condition(s).

To the best of our knowledge, this is the first study assessing how patients with concomitant chronic conditions are excluded from ongoing RCTs assessing interventions for patients with common chronic conditions. Other studies evaluated exclusion criteria in published RCTs. Van Spall $e t a l^{14}$ reviewed published RCTs to determine the nature and extent of exclusion criteria: medical comorbidities (acute and chronic conditions) were excluded in $81 \%$ of published RCTs. Jadad et $a l^{15}$ analysed reports of RCTs assessing interventions for patients with chronic conditions published from 1995 to 2010 and found patients with multimorbidity excluded in $65 \%$ of published trials. Our results agree with Smith et $a l,{ }^{16}$ who performed a systematic review of all interventional studies assessing interventions specifically directed towards patients defined as multimorbid. The authors retrieved only 10 trials assessing a range of complex interventions.

Our study shows that primary research used to develop guidelines for chronic conditions frequently excludes patients with concomitant chronic conditions, ${ }^{14}$ so the external validity or generalisability of much of the current evidence is relatively weak. ${ }^{17}$ Every individual recommendation included in a guideline may be rational and evidence-based, but the sum of all recommendations for patients with multimorbidity may not be. ${ }^{11}$ Indeed, the inappropriate generalisation of trials results may lead to unintended harm. Research is particularly needed on the clustering of conditions in patients with multimorbidity. ${ }^{18}$ Few trials are specifically targeting patients with concomitant chronic conditions.

Our study has some limitations. First, we focused on only ongoing trials registered at ClinicalTrials.gov. Although ClinicalTrials.gov is the most commonly used register, ${ }^{19}$ these records may be not representative of all ongoing trial records. Second, our results relied on exclusion criteria listed in trial records at ClinicalTrials. gov, which may not be complete or may have changed. However, protocols are poorly accessible ${ }^{20}$ and trial registries are frequently used to assess the trial information. ${ }^{21}$ 


\begin{tabular}{|c|c|c|c|c|c|c|c|c|c|c|c|}
\hline Characteristics & $\begin{array}{l}\text { All } \\
n=319\end{array}$ & $\begin{array}{l}\text { CHD } \\
\mathrm{n}=36\end{array}$ & $\begin{array}{l}\text { Hypertension } \\
\mathrm{n}=38\end{array}$ & $\begin{array}{l}\text { Heart } \\
\text { failure } \\
n=36\end{array}$ & $\begin{array}{l}\text { Stroke/ } \\
\text { TIA } \\
\mathbf{n}=22\end{array}$ & $\begin{array}{l}\text { Atrial } \\
\text { fibrillation } \\
n=25\end{array}$ & $\begin{array}{l}\text { Diabetes } \\
\mathrm{n}=109\end{array}$ & $\begin{array}{l}\text { COPD } \\
n=34\end{array}$ & $\begin{array}{l}\text { Chronic } \\
\text { pain } \\
n=12\end{array}$ & $\begin{array}{l}\text { Depression } \\
\mathrm{n}=40\end{array}$ & $\begin{array}{l}\text { Dementia } \\
\mathrm{n}=7\end{array}$ \\
\hline $\begin{array}{l}\text { Trials excluding at least } \\
\text { one chronic condition }\end{array}$ & $251(79)$ & $25(69)$ & $33(87)$ & $30(83)$ & $17(77)$ & $20(80)$ & $87(80)$ & $28(82)$ & $11(92)$ & $30(75)$ & $6(86)$ \\
\hline \multicolumn{12}{|l|}{ Excluded conditions } \\
\hline $\mathrm{CHD}$ & $47(15)$ & - & $10(26)$ & $1(3)$ & $0(0)$ & $1(4)$ & $16(15)$ & $13(38)$ & $0(0)$ & $5(13)$ & $4(57)$ \\
\hline Hypertension & $44(14)$ & $4(11)$ & - & $3(8)$ & $2(9)$ & $4(16)$ & $18(17)$ & $10(29)$ & $0(0)$ & $4(10)$ & $3(43)$ \\
\hline Heart failure & $92(29)$ & $8(22)$ & $15(40)$ & - & $0(0)$ & $4(16)$ & $44(40)$ & $20(59)$ & $0(0)$ & $4(10)$ & $3(43)$ \\
\hline Stroke/TIA & 37 (12) & $1(3)$ & $9(24)$ & $1(3)$ & - & $1(4)$ & $11(10)$ & $7(21)$ & $1(8)$ & $4(10)$ & $4(57)$ \\
\hline Atrial fibrillation & $34(11)$ & $1(3)$ & $9(24)$ & $2(6)$ & $1(5)$ & - & $10(9)$ & $10(30)$ & $0(0)$ & $4(10)$ & 2 (29) \\
\hline Diabetes & $23(7)$ & $1(3)$ & $9(24)$ & $0(0)$ & $1(5)$ & $0(0)$ & - & 7 (21) & $1(8)$ & $4(10)$ & $0(0)$ \\
\hline COPD & $6(2)$ & $1(3)$ & $1(3)$ & $3(8)$ & $0(0)$ & $1(4)$ & $1(1)$ & - & $0(0)$ & $0(0)$ & $0(0)$ \\
\hline Chronic pain & $2(1)$ & $0(0)$ & $0(0)$ & $0(0)$ & $1(5)$ & $0(0)$ & $1(1)$ & $0(0)$ & - & $0(0)$ & $0(0)$ \\
\hline Depression & 53 (17) & $3(8)$ & 7 (18) & $3(8)$ & $6(27)$ & $3(12)$ & $14(13)$ & $9(27)$ & $7(58)$ & - & $3(43)$ \\
\hline Dementia & $24(8)$ & $0(0)$ & $2(5)$ & $3(8)$ & $4(18)$ & $0(0)$ & $10(9)$ & $2(6)$ & $0(0)$ & $5(13)$ & - \\
\hline Other(s) & $86(27)$ & 12 (33) & $11(29)$ & 17 (47) & $6(27)$ & 9 (36) & $25(23)$ & $4(12)$ & $4(33)$ & $19(48)$ & $1(14)$ \\
\hline $\begin{array}{l}\text { No. of excluded } \\
\text { conditions, median } \\
\text { (Q1-Q3) }\end{array}$ & $9(6-14)$ & $11(7-12)$ & $12(6-21)$ & $10(6-13)$ & $8(5-9)$ & $9(7-13)$ & $8(6-13)$ & $13(7-21)$ & $10(6-13)$ & $8(4-13)$ & $10(8-14)$ \\
\hline $\begin{array}{l}\text { Trials targeting patients } \\
\text { with multiple chronic } \\
\text { conditions }\end{array}$ & $37(12)$ & $11(31)$ & $12(32)$ & 7 (19) & $1(5)$ & 8 (32) & $14(13)$ & $1(3)$ & $1(8)$ & 7 (18) & $1(14)$ \\
\hline
\end{tabular}


Figure 2 Flow diagram of trial records' eligibility criteria.

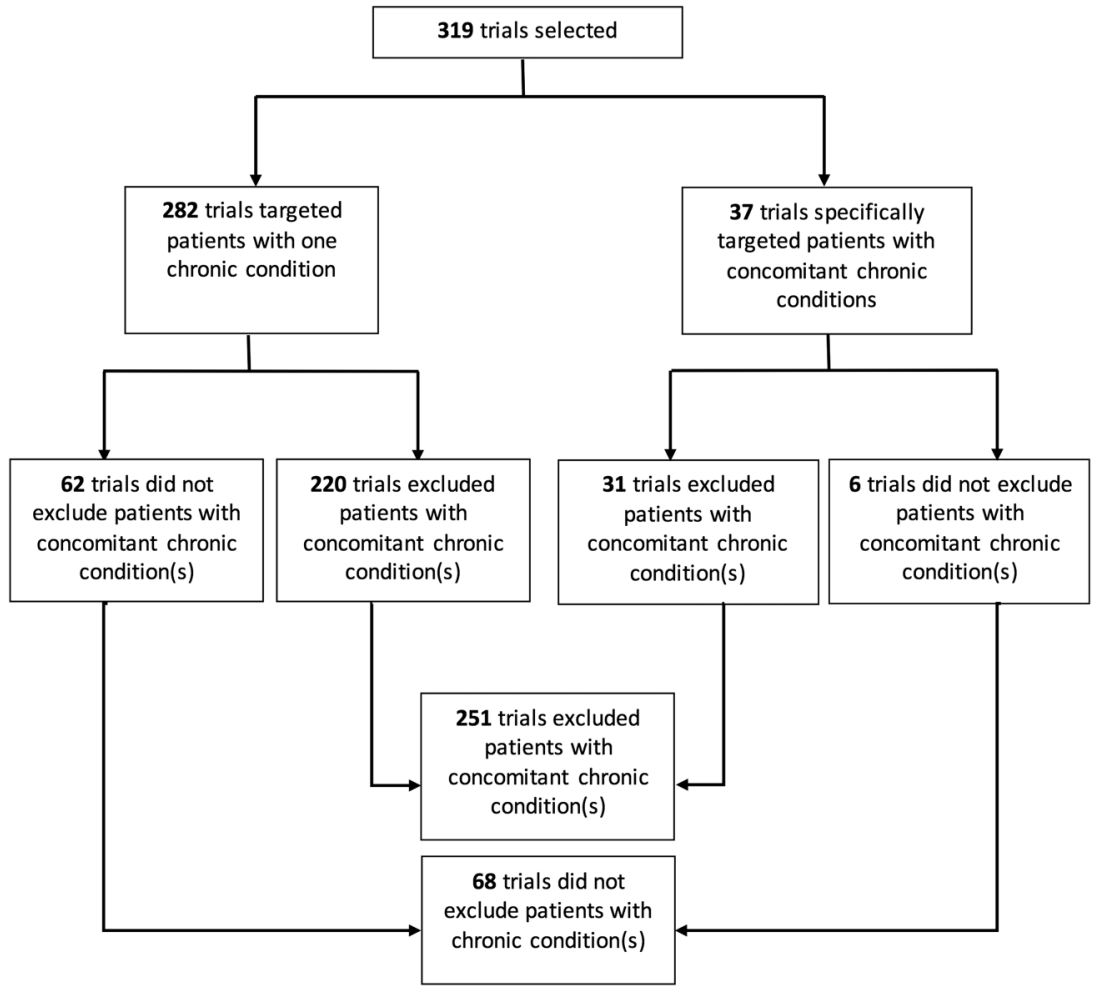

Figure 3 Proportion of trials excluding patients with concomitant chronic condition(s) by prevalence of concomitant chronic conditions. For example, $91 \%$ of patients with coronary heart disease (CHD) had a concomitant chronic condition, but 25 trials $(69 \%)$ targeting patients with $\mathrm{CHD}$ excluded patients with concomitant chronic condition(s).

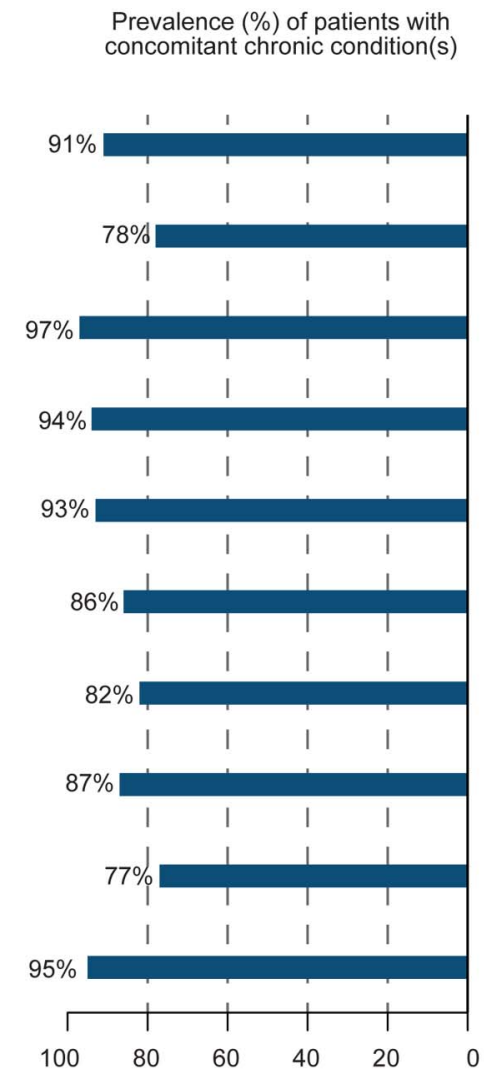

Proportion (\%) of trials excluding patients with concomitant chronic condition(s)

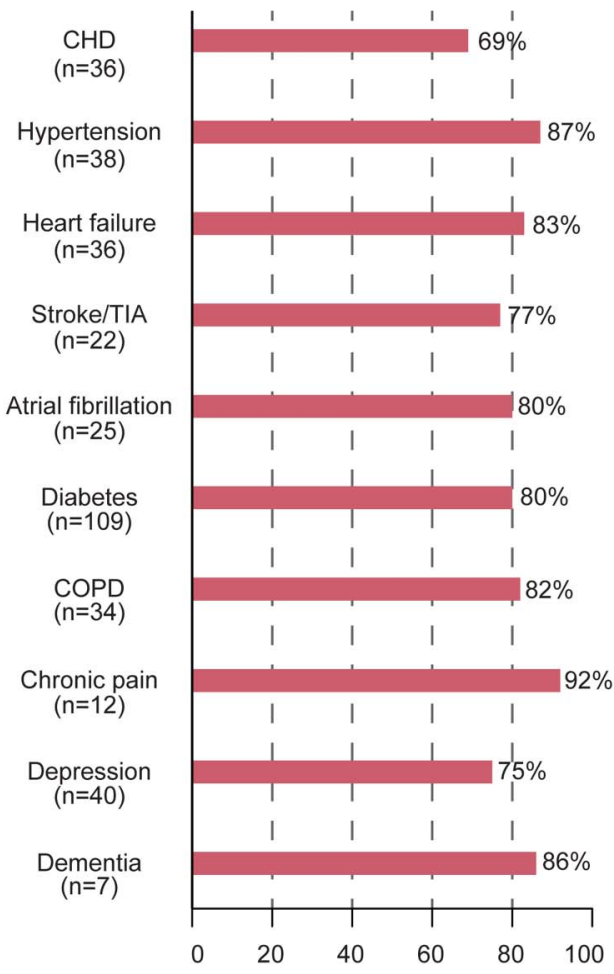

Thus, our results may underestimate the prevalence of excluding patients with multiple chronic conditions ${ }^{22}$ from trial results with protocols. Third, we did not assess the characteristics of the patients actually included in these trials. Some patients with concomitant chronic conditions may be excluded by the investigators even if no exclusion criterion was listed in the protocol. Fourth, the UK prevalence estimates we used to balance our results may not be relevant for all ongoing trial records included. 


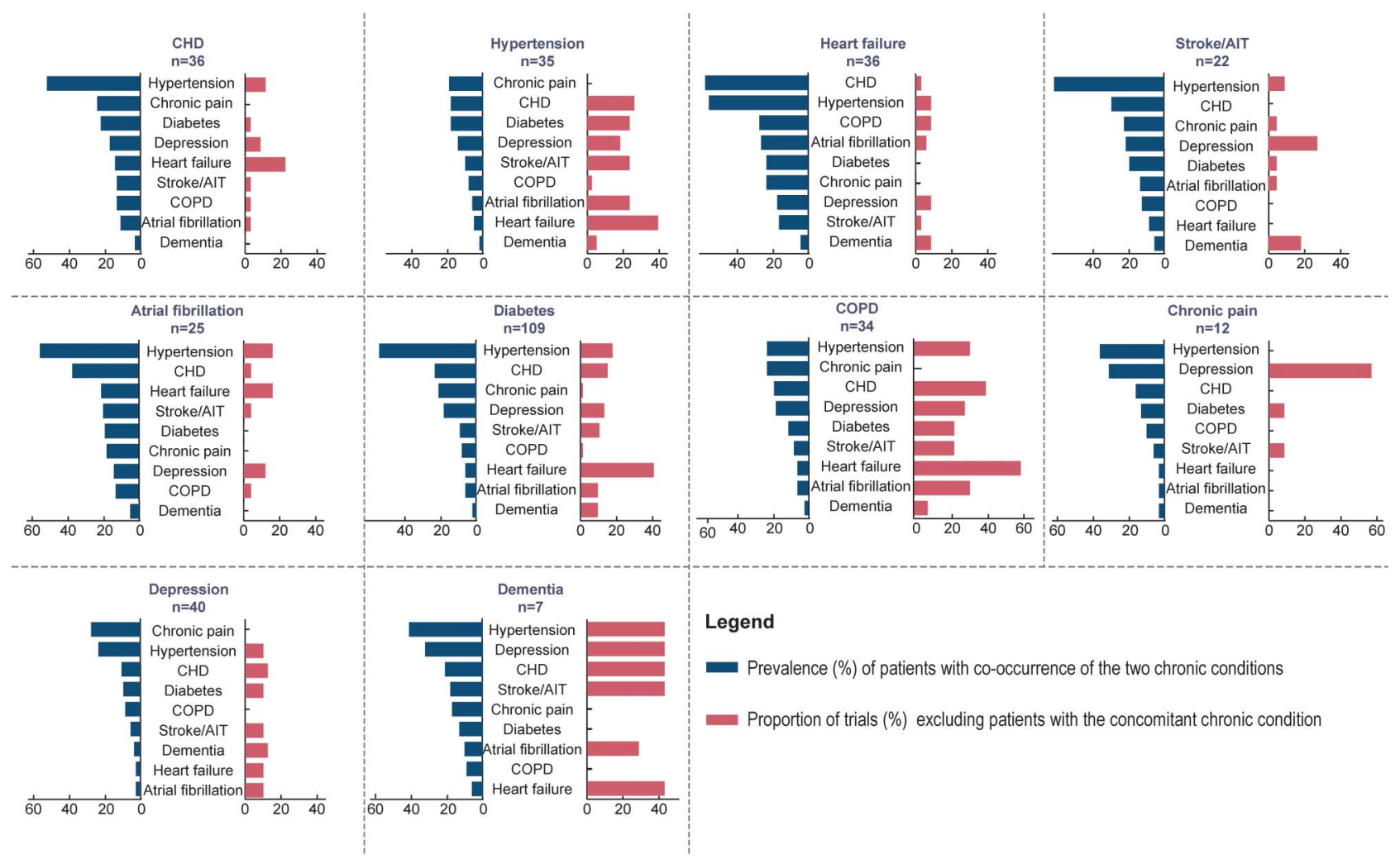

Figure 4 For each chronic condition targeted, the proportion of trials excluding patients with 1 of the 10 common chronic conditions selected according to the prevalence of the associations. For example, $61 \%$ of patients with stroke or transient ischaemic attack (TIA) also had hypertension, but $9 \%$ of the trials targeting patients with stroke/TIA excluded patients with hypertension.

Chronic conditions and multimorbidity are becoming the greatest epidemic in high-income countries. Management of multimorbid patients is challenging, particularly because clinical practice guidelines are developed in silo for a single disease. ${ }^{23}$ Physicians are supposed to combine these recommendations when caring for patients with multimorbidity, which can be harmful and burdensome for patients. ${ }^{7}{ }^{10}$ Some researchers have called for new forms of clinical practice guidelines and evidence summaries for the management of multimorbidity. ${ }^{11}$

Our study highlights the need for research of patients with multimorbidity. Assessing the impact of interventions for all associations may not be realistic. However, a clear research agenda on prevalent associations of chronic conditions to produce evidence for caring for these patients is needed. Researchers should also pay attention to eligibility criteria when designing a trial: they should try not to exclude concomitant chronic conditions, so as to be more representative of the real-world population. Finally, our findings may raise a conceptual issue related to the funding of trials: as for guidelines, funding resources are also obtained in silo, condition by condition. Thus, finding resources to finance a trial assessing an intervention for patients with multiple chronic conditions may be difficult.

\section{Conclusion}

Despite the high prevalence of multimorbidity, more than three-quarters of ongoing research registered at ClinicalTrials.gov and assessing interventions for patients with chronic health conditions excluded patients with concomitant chronic conditions. This finding could lead to a questionable generalisability of these results to the real-world population. Primary care research should be conducted according to the prevalence of multimorbidity in the real world. The research agenda should be revised to better account for the prevalence of concomitant chronic conditions.

\section{Author affiliations}

${ }^{1}$ Faculté de Médecine, Département de médecine générale, Université Paris Descartes, Sorbonne Paris Cité, Paris, France

${ }^{2}$ Faculté de Médecine, Université Paris Descartes, Sorbonne Paris Cité, Paris, France

${ }^{3}$ METHODS Team, Epidemiology and Statistics Sorbonne Paris Cité Research Center UMR 1153, INSERM, Paris, France

${ }^{4}$ Assistance Publique des Hôpitaux de Paris (AP-HP), Hôpital Hôtel Dieu, Centre d'Epidémiologie Clinique, Paris, France

${ }^{5}$ French Cochrane Center, Paris, France

${ }^{6}$ Department of Epidemiology, Columbia University Mailman School of Public Health, New York, New York, USA

Acknowledgements The authors thank Elise Diard for the graphs and Laura Smales for critical reading and English correction of the manuscript. 
Contributors All authors participated in the conception and design of the study. CBdV and CB performed the search, selected the trials and extracted data. CBdV performed the statistical analysis. All authors interpreted the results. CBdV drafted the manuscript and all authors revised the manuscript critically for important intellectual content. All authors read and approved the final version of the manuscript.

Funding This research received no specific grant from any funding agency in the public, commercial or not-for-profit sectors.

Competing interests None declared.

Provenance and peer review Not commissioned; externally peer reviewed.

Data sharing statement No additional data are available.

Open Access This is an Open Access article distributed in accordance with the Creative Commons Attribution Non Commercial (CC BY-NC 4.0) license, which permits others to distribute, remix, adapt, build upon this work noncommercially, and license their derivative works on different terms, provided the original work is properly cited and the use is non-commercial. See: http:// creativecommons.org/licenses/by-nc/4.0/

\section{REFERENCES}

1. Bauer UE, Briss PA, Goodman RA, et al. Prevention of chronic disease in the 21st century: elimination of the leading preventable causes of premature death and disability in the USA. Lancet 2014;384:45-52.

2. Alwan A. Global status report on noncommunicable diseases 2010. World Health Organization, 2011.

3. Salisbury C, Johnson L, Purdy S, et al. Epidemiology and impact of multimorbidity in primary care: a retrospective cohort study. Br J Gen Pract 2011;61:e12-21.

4. Ward BW, Schiller JS, Goodman RA. Multiple chronic conditions among US adults: a 2012 update. Prev Chronic Dis 2014;11:4-7.

5. Barnett K, Mercer SW, Norbury M, et al. Epidemiology of multimorbidity and implications for health care, research, and medical education: a cross-sectional study. Lancet 2012;380:37-43.

6. Guthrie B, Payne K, Alderson P, et al. Adapting clinical guidelines to take account of multimorbidity. BMJ 2012;345:e6341.

7. Lugtenberg M, Burgers JS, Clancy C, et al. Current guidelines have limited applicability to patients with comorbid conditions: a systematic analysis of evidence-based guidelines. PLOS ONE 2011;6:e25987.
8. Tinetti ME, McAvay G, Trentalange M, et al. Association between guideline recommended drugs and death in older adults with multiple chronic conditions: population based cohort study. BMJ 2015;35:h4984.

9. Guthrie B, Makubate B, Hernandez-Santiago V, et al. The rising tide of polypharmacy and drug-drug interactions: population database analysis 1995-2010. BMC Med 2015;13:74.

10. Dumbreck S, Flynn A, Nairn M, et al. Drug-disease and drug-drug interactions: systematic examination of recommendations in 12 UK national clinical guidelines. BMJ 2015;350:h949.

11. Wallace E, Salisbury C, Guthrie B, et al. Managing patients with multimorbidity in primary care. BMJ 2015;350:h176.

12. Gandhi GY, Murad MH, Fujiyoshi A, et al. Patient-important outcomes in registered diabetes trials. JAMA 2008;299:2543-9.

13. Pino C, Boutron I, Ravaud P. Outcomes in registered, ongoing randomized controlled trials of patient education. PLOS ONE 2012;7:8-11.

14. Van Spall HGC, Toren A, Kiss A, et al. Eligibility criteria of randomized controlled trials published in high-impact general medical journals: a systematic sampling review. JAMA 2007:297:1233-40.

15. Jadad AR, To MJ, Emara M, et al. Consideration of multiple chronic diseases in randomized controlled trials. JAMA 2011;306:2670-2.

16. Smith SM, Wallace E, O'Dowd T, et al. Interventions for improving outcomes in patients with multimorbidity in primary care and community settings. Cochrane Database Syst Rev 2016;3: CD006560.

17. Rothwell PM. Factors that can affect the external validity of randomised controlled trials. PLoS Clin Trials 2006:1:e9.

18. Violan C, Foguet-Boreu Q, Flores-Mateo G, et al. Prevalence, determinants and patterns of multimorbidity in primary care: a systematic review of observational studies. PLOS ONE 2014;9: $3-11$.

19. Dickersin K, Rennie D. The evolution of trial registries and their use to assess the clinical trial enterprise. JAMA 2012;307:1861-4.

20. Odutayo A, Altman DG, Hopewell S, et al. Reporting of a publicly accessible protocol and its association with positive study findings in cardiovascular trials (from the Epidemiological Study of Randomized Trials [ESORT]). Am J Cardiol 2015;116:1280-3.

21. Goldacre B, Drysdale H, Powell-Smith A, et al. The COMPare Trials Project. 2016

22. Blümle A, Meerpohl JJ, Rucker G, et al. Reporting of eligibility criteria of randomised trials: cohort study comparing trial protocols with subsequent articles. BMJ 2011;342:d1828.

23. Boyd CM, Darer J, Boult C, et al. Clinical practice guidelines and quality of care for older patients. JAMA 2005;294:716-24. 